Im „Journal Club“ werden Originalarbeiten aus der internationalen Fachliteratur referiert und kommentiert.

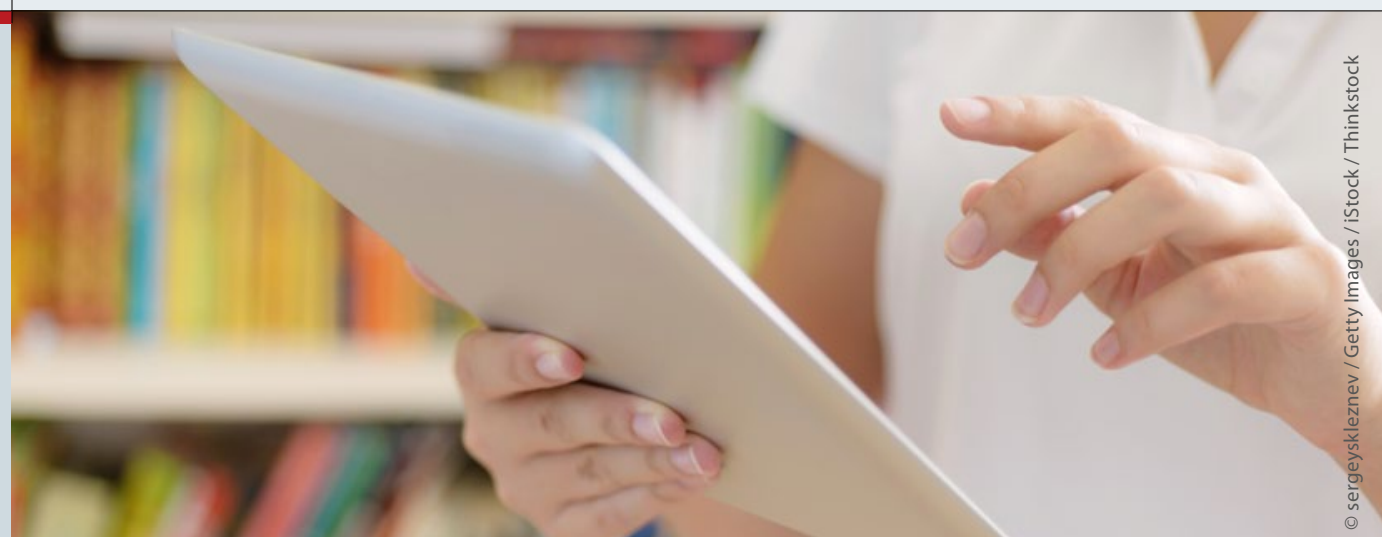

\section{Sport schützt wahrscheinlich auch vor Gebärmutterkrebs}

Gut jedes fünfte Endometriumkarzinom ließe sich durch mehr körperliche Aktivität verhindern, so das Ergebnis einer prospektiven Kohortenstudie aus Norwegen.

$\mathrm{J}$ e mehr sie sich körperlich betätigten, desto seltener erkrankten die Teilnehmerinnen der NOWAC-Studie (Norwegian Women and Cancer) am Endometriumkarzinom. Das ist die Quintessenz der prospektiven Kohortenstudie, an der 52.370 Frauen im Alter zwischen 30 und 70 Jahren beteiligt waren. Das mittlere Alter zu Studienbeginn lag bei 51 Jahren. Über einen Zeitraum von im Mittel knapp 13 Jahren wurde bei 687 Teilnehmerinnen ein Gebärmutterkrebs diagnostiziert. Dieser stellte sich bei $83,8 \%$ als östrogenabhängiger Tumor vom Typ 1 heraus. Kristin B. Borch von der Arctic University of Norway in Tromsø und ihr Team hatten die Frauen zu zwei Zeitpunkten zu ihrer körperlichen Aktivität befragt: einmal zu Studienbeginn und ein zweites Mal sechs bis acht Jahre später. Dabei zählte nicht nur Freizeitsport, sondern auch Bewegung im Haushalt, in der Arbeit oder unterwegs.

\section{Erkrankungsrisiko erhöht}

Als Referenz diente der Bereich von 5 bis 6 auf der Skala. In diesen fielen 43\% der Frauen. $74 \%$ waren ebenso aktiv oder noch aktiver. Wie Borch und Kollegen berichten, hatten Frauen auf dem Level 1 bis 2 ein um 60\% höheres Erkrankungsrisiko als die Teilnehmerinnen in der Referenzgruppe. Auf dem höchsten Level (9 bis 10) war das Risiko um relative $27 \%$ erniedrigt. Bei Werten von 4 und darunter, so errechneten die Autoren, könnten 21,9\% der Endometrium- karzinomfälle dadurch vermieden werden, dass die Frauen ihre Aktivität auf ein Level von 5 oder mehr steigerten. In ihrem Rechenmodell hatten die Forscher sowohl BMI, Rauchen und Alkoholkonsum als auch Beginn der Menarche, Zahl der Geburten, Menopausenstatus, Einnahme von Kontrazeptiva pie (HET) berücksichtigt.

\section{Effekt hängt nicht unbedingt vom BMI ab}

Da der BMI gemeinhin als starker Risikofaktor für die Entwicklung von Gebärmutterhalskrebs gilt, hatten Borch und ihr Team beschlossen, den Zusammenhang zur körperlichen Aktivität in drei unterschiedlichen Gruppen zu untersuchen: bei normalgewichtigen, überund eine eventuelle Hormonersatzthera- gewichtigen und adipösen Frauen. Hier zeigte sich, dass ein niedriges Aktivitätslevel nur in der Gruppe der adipösen Frauen signifikant mit dem Erkrankungsrisiko verknüpft war. Allerdings war der Interaktionstest für die beiden Variablen nicht signifikant.

Ob es an dem Einfluss der Bewegung auf die endogenen Geschlechtshormonspiegel liegt oder ob der Effekt möglicherweise insulinvermittelt ist - beides stellen die Forscher zur Debatte -, wird man noch herausfinden müssen. Letztlich, so die Forscher, habe die Studie bestätigt, dass mangelnde Bewegung einen unabhängigen Risikofaktor für die Erkrankung am Gebärmutterhalskrebs darstelle. Der BMI hatte das Ergebnis offenbar nicht wesentlich beeinflusst. Borch und ihr Team empfehlen jedoch, die Ergebnisse zurückhaltend zu interpretieren; insbesondere könne man aus der Studie keine Empfehlung für ein bestimmtes Maß an Aktivität ableiten. (eo)

Borch KB et al. Physical activity and risk of endometrial cancer in the Norwegian Women and Cancer (NOWAC) study. Int J Cancer 2017; online 20. Januar. doi: 10.1002/ijc.30610
Hier steht eine Anzeige.

Springer 